\title{
Antifungal activity of chitosan against Fusarium oxysporum f. sp. cubense.
}

\begin{abstract}
The in vitro antifungal activity of chitosan against Fusarium oxysporum f. sp. cubense Race 4 (FocR4) the causal agent of banana wilt was investigated. Chitosan at all concentrations tested reduced the hyphal growth of FocR4 on potato dextrose agar media and recording maximum inhibition of $76.36 \%$ at $8 \mathrm{mg} / \mathrm{mL}$. The inhibitory effect was found to increase as chitosan concentration increases. The $50 \%$ effective concentration value was estimated by probit analysis, and it was $1.4 \mathrm{mg} / \mathrm{mL}$. Chitosan was more effective in potato dextrose broth where it completely inhibited the mycelial growth of FocR4 at all concentrations tested. Chitosan inhibited the sporulation of FocR 4 by a maximum of $96.53 \%$ at $8 \mathrm{mg} / \mathrm{mL}$ chitosan, and $100 \%$ inhibition for spore germination was recorded at all concentrations tested. Chitosan at concentrations of more than $1.6 \mathrm{mg} / \mathrm{mL}$ was also found to induce morphological changes in FocR4 characterized by agglomeration of hyphae, abnormal shapes, vesicles, or empty cells devoid of cytoplasm in the mycelia.
\end{abstract}

Keyword: Chitosan; Fusarium oxysporum; Antifungal; Banana wilt. 